\title{
Survival score scales of patients operated with spinal metastases: retrospective application in a Brazilian population
}

\author{
Escalas de prognóstico em pacientes operados com metástases vertebrais: aplicação \\ retrospectiva em população brasileira
}

Eduardo Carvalhal Ribas', Luis Roberto Mathias Junior', Vinicius Monteiro Guirado', Roger Schmidt Brock', Mario Augusto Taricco', Mauro Miguel Daniel², Rafael Burgomeister Lourenço², Manoel Jacobsen Teixeira'

\begin{abstract}
Spinal cord epidural metastasis (SEM) is a common complication of systemic cancer. Predicting these patient's survival is a key factor to select the proper treatment modality, but the three most used score scales to predict their survival (Tokuhashi revised score, Tomita score and Bauer modified score) were designed in single institutions and their reliability to predict correctly the patient's survival were first tested only in those specific populations. This prognostication issue is addressed in this article, evaluating retrospectively the survival of 17 patients with SEM from a Brazilian general hospital with these score scales. Our results show that the actual survival of those patients were worse than the predicted of all three score scales, suggesting that differences between the different populations might have affected their reliability and alert that their usage as a major factor to select the most appropriate treatment have to be done with caution.
\end{abstract}

Keywords: spinal metastasis, score scales, prognosis, neurosurgery, surgical treatment.

RESUMO

Metástases vertebrais são uma complicação comum em pacientes com câncer sistêmico. Avaliar o prognóstico e a sobrevida desses pacientes é um fator de grande importância para escolher o tratamento mais adequado, porém as três escalas mais usadas atualmente para prever a sobrevida deles (Tokuhashi revisada, Tomita e Bauer modificada) foram desenhadas em instituições isoladas, e sua habilidade em estimar corretamente a sobrevida desses pacientes foram testadas primeiramente apenas nessas populações específicas. Essa questão de estimar o prognóstico é abordada nesse artigo, analisando retrospectivamente a sobrevida de 17 pacientes com metástase vertebral provenientes de um hospital geral no Brasil com essas escalas. Nossos resultados apontam que a sobrevida real desses pacientes foi menor que a prevista pelas três escalas, sugerindo que as diferenças entres as diferentes populações podem ter afetado a aplicabilidade delas. Assim, alertamos que o uso dessas escalas em populações diferentes das estudadas originalmente deve ser feito com cuidado.

Palavras-chave: metástases vertebrais, escalas de prognóstico, neurocirurgia, tratamento cirúrgico.

Spinal cord epidural metastases (SEM) are a common complication of systemic cancer, and has an increasing incidence in oncological patients ${ }^{1}$. From the vertebral body, these metastases can invade into the vertebral canal and cause spinal cord compression and vascular insufficiency, leading to myelopathy, cauda equina, or root dysfunction syndromes. Pain is the most common symptom, present in $83-95 \%$ of these patients ${ }^{1}$. Also, two thirds of them have motor signs at the time of diagnosis and sensory deficits can be detectable in their majority ${ }^{2}$. Bowel and bladder disturbances tend to appear later, but about half of patients with SEM are already catheter dependent at diagnosis ${ }^{1}$.
Unfortunately, SEM is part of an already spread cancer disease and its treatment should focus on improving the patient's quality of life ${ }^{3}$. Several protocols and strategies about how to treat patients with SEM, including surgery, radiotherapy and chemotherapy have been studied but the debate is still open on which are the patients who will benefit more from each treatment. Surgical treatment can lead to accurate diagnosis, control refractory pain to non-operative measures, preserve or restore neurological function, and maintain alignment and stability of the bony spine ${ }^{4}$, but it is also accepted that patients with limited prognosis should not be candidates

${ }^{1}$ Universidade de São Paulo, Hospital das Clínicas, Departamento de Neurologia, Disciplina de Neurocirurgia, Sao Paulo SP, Brazil;

2Universidade de São Paulo, Hospital das Clínicas, Departamento de Radiologia, Sao Paulo SP, Brazil;

Correspondence: Eduardo Carvalhal Ribas; Rua Professor Eduardo Monteiro, 567; 05614-120 São Paulo SP, Brasil; E-mail: eribas@gmail.com

Conflict of interest: There is no conflict of interest to declare.

Received 06 April 2014; Received in final form 08 September 2015; Accepted 28 September 2015. 
to surgery. Some authors state a minimum of 3-month life expectancy to consider operative treatment ${ }^{5}$, and others extend this limit to about 6 months ${ }^{4,6}$. Therefore, predicting survival is the key factor in selecting the proper treatment modality ${ }^{6,7}$.

There are three leading scales used to predict the prognosis of the patients with SEM: the Tokuhashi revised score ${ }^{6}$, the Tomita score ${ }^{4}$ and the Bauer modified score ${ }^{8}$. Each one was designed in a single institution and their reliability to predict the prognosis was first tested in that specific population: Tokuhashi $^{6}$ carried his studies in Tokyo, Japan; the Tomita score $^{4}$ was tested in Kanazawa, Japan, a much smaller city; and the Bauer modified scoring system ${ }^{9}$ was designed in Graz, Austria. Many differences can be found between different populations, and their application in different situations can possibly predict patient survival wrongly. Also, these score scales differ greatly in the kind of parameters assessed and the importance of these factors in the total score, resulting in different survival predictions for the same patient and contradictory treatment strategies suggestions between them ${ }^{8}$.

The objective of this study was to compare the predicted survival time of these score systems to the actual survival time observed of each patient and to discover if they could be applied to the Brazilian population with good reliability, to be used later to guide patients to the most appropriate treatment option.

\section{METHOD}

We retrospectively analyzed 24 consecutive patients operated between July/2008 and March/2010 in the Hospital das Clíncias of Universidade de São Paulo, Brazil, due to SEM by our neurosurgical staff. Our inclusion criteria included only adult patients (above 18 years old), patients submitted to neurosurgery due to their neurological presentation related to SEM, patients operated in our institution and by our neurosurgical staff, patients with complete radiological investigation, and patients who agreed to participate in the study after it was explained to them. We excluded from our study pediatric patients, patients operated in other institution or by other neurosurgeons, with incomplete radiological investigation and patients not willing to participate in the study.

The decision to operate each patient, and the operative technique, was made independently by our neurosurgical staff and not using any score scale as a protocol. Our surgeries were addressed to partially (or sub-totally) remove the tumor and decompress neurological structures, in order to reestablish neurological function and alleviate pain. Vertebral fixation and stabilization was made only if signs of spinal instability were noted.

Overall survival was set as primary outcome, and follow-up was made by ambulatory consultation and telephone calls until May/2011, when most patients had already died and four were alive for at least one year after surgery.
All patients were submitted to thoracic and abdominal CT scan to screen for other metastases, and bone scan was made if bone metastases were suspected, before surgery or during follow-up if there was not a complete radiological screening before operation because of the emergency need in some cases. Seven patients (29\%) were lost during follow-up and, finally, all necessary information to complete the three score systems about 17 patients could be noted and is presented in this study.

Our results are finally reported using descriptive statistical analysis due to small number of patients enrolled.

\section{RESULTS}

Seventeen patients were followed-up by a mean period of 8.85 months (range: 1-27). At the end of follow-up, 13 (76\%) patients had already died with a mean actual survival time of 5.03 months (range: 1-27 months) and four patients were still alive, all of them for more than one year after surgery.

The majority of patients were male (83\%) and 3 were female, with a mean age of 65 years (range: 29-77). Histological analysis of the metastases revealed seven different primary cancer sites, with prostate as the most prevalent (35\%) (Table 1). All patients had spine cord compression symptoms at the time of surgery, where three patients (18\%) were operated due to cervical metastases, 10 patients (58\%) due to thoracic metastases, 1 patient $(6 \%)$ with a thoraco-lumbar transition lesion and 3 patients $(18 \%)$ due to lumbar metastases. Surgical technique was chosen independently without a protocol, resulting in 58\% of surgeries performed only to decompress the spinal cord and not aiming to radically resect the tumor. The most important clinical information, scores and survival of each patient are present in Tables 2 and 3.

\section{Tokuhashi revised score scale}

Tokuhashi et al. ${ }^{10}$ first published his score in 1990 and later revised $\mathrm{it}^{6,11}$, subdividing the primary cancer histology in five categories. The Tokuhashi Revised Score ${ }^{6}$, used in this study, takes six variables into account: general medical condition, number of extra spinal metastases, number of vertebral metastases, visceral metastases, primary tumor type, and presence of neurologic deficits. Each parameter is evaluated with a score from 0 to 2 points, but the primary tumor type, which varies from 0 to 5 points. The final score, given by all points, ranges from 0 to 15 (Table 1).

Two patients had 12 points or more, occupying the better score range with a predicted survival longer than one year, but none of them reached this survival time during follow-up (mean actual survival of only 5 months). Seven patients were in the middle score range, with 9 to 11 points and a predicted survival interval of 6 to 12 . One of these patients (14\%) survived only 1.5 months, another patient (14\%) had an actual survival of 8 months and $5(71 \%)$ lived for more than one year. 
Table 1. Comparison between three three scales used to predict the prognosis of the patients with spinal cord epidural metastasis: Tokuhashi Revised Scoring System, Tomita Scoring System, Bauer Modified Scoring Sys.

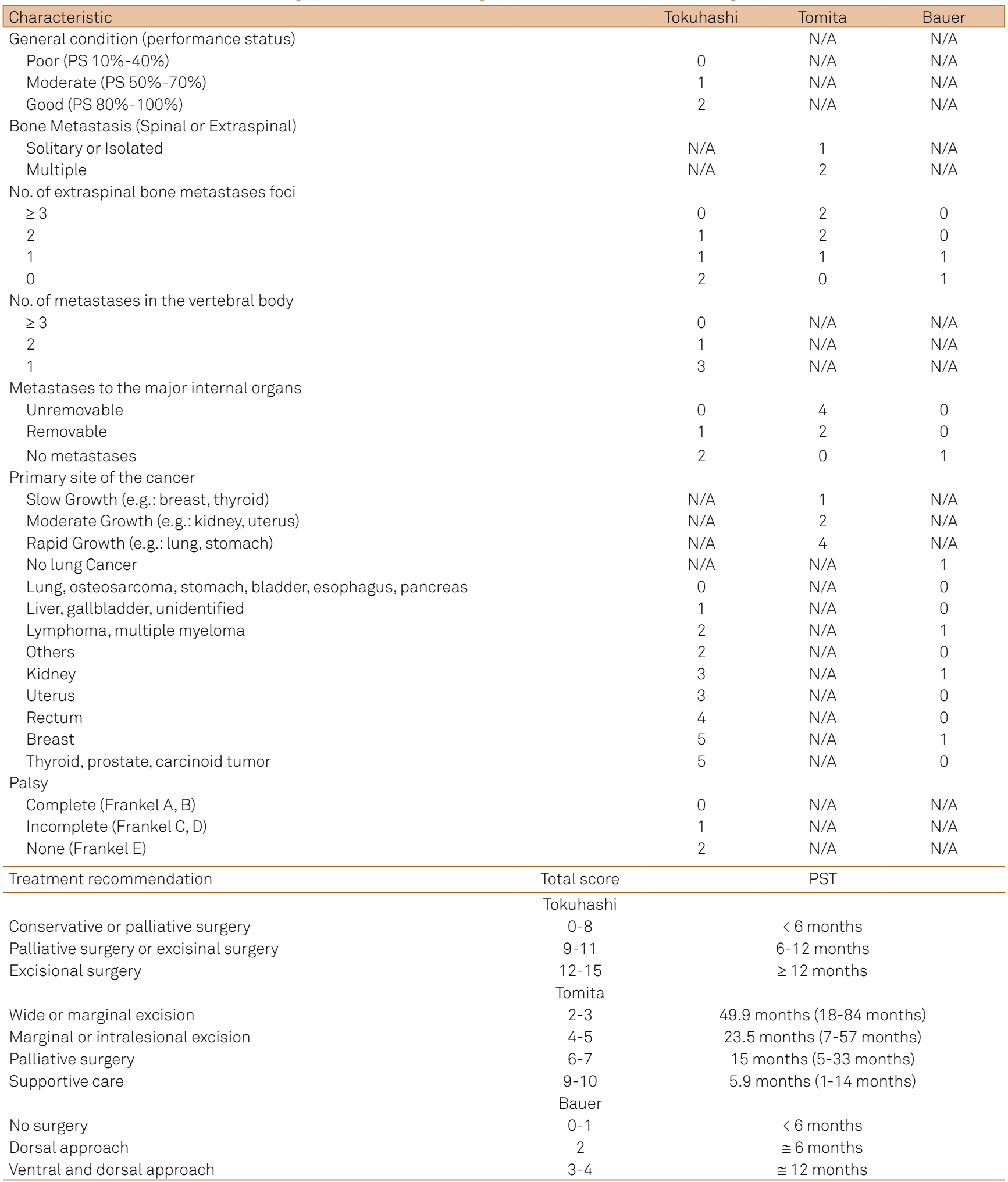

N/A: Non Applicable; PST: Predicted Survival Time (estimated for the Bauer Modified Scoring System).

Eight patients (47\%) had 8 or fewer points in this score, and according to the original authors they would have a short survival. Six of these patients (75\%) actually died before 6 months and the other $2(25 \%)$ survived 9 and 10 months each (mean actual survival of 4 months).
The accuracy of this score to predict survival longer than 12 months, between 6 to 12 months or less than 6 months was $0 \%, 14 \%$ and $75 \%$, respectively. Overall, the Tokuhashi Revised Score was correct in predicting the survival in only 7 of 17 patients (41\%). 
Table 2. Patients' clinical, oncological, radiological and surgical information.

\begin{tabular}{|c|c|c|c|c|c|c|c|c|c|}
\hline & Patient & Sex & Age & $\begin{array}{c}\text { Primary } \\
\text { Cancer Site }\end{array}$ & Clinical Info & Radiological Info & $\begin{array}{l}\text { SEM } \\
\text { level }\end{array}$ & $\begin{array}{l}\text { Performed } \\
\text { Surgery }\end{array}$ & $\begin{array}{l}\text { Surgery } \\
\text { Date }\end{array}$ \\
\hline 1 & IDSF & $\mathrm{F}$ & 66 & Thyroid & rankel E & OM, > 3 ESM, > 3 VRM & $\mathrm{C} 4, \mathrm{C} 5$ & & 07/14/2008 \\
\hline 2 & JBS & M & o & Kidney & Moderate KPS, Frankel C & NTVM, SOM, None ESM, One VRM & & & \\
\hline 3 & $J M C L$ & $\mathrm{~F}$ & 65 & Thyroid & Moderate KPS, Frankel C & None VIM, SOM, None ESM, Two VRM & L3, L4, L5 & Desc + PTR + PS & $09 / 26 / 2008$ \\
\hline 4 & CYM & M & 58 & Liver & Moderate KPS, Frankel D & None VIM, SOM, None ESM, One VRM & T6, Т7 & Desc + PS & $10 / 06 / 2008$ \\
\hline 5 & APRA & M & 77 & Colorectal & Moderate KPS, Frankel E & NTVM, SOM, None ESM, None VRM & T3 & Desc & \\
\hline 6 & SLF & M & 62 & & Moderate & None VIM, I & & & \\
\hline 7 & & M & 72 & & & & & & \\
\hline 8 & LACN & M & 76 & dney & Moderate KPS, Frankel D & NTVM, SOM, N & & Desc + P & \\
\hline 9 & $\mathrm{RCL}$ & M & 29 & & kel C & & C & PS & \\
\hline 10 & MSS & $\mathrm{F}$ & 62 & hyroid & Moderate KPS, & NTVM, SOM, None ESM, None VRM & & Desc + PTR + PS & \\
\hline 11 & & M & 79 & & & None VIM, SOM, None ESM, None VRM & & & \\
\hline 12 & JJP & M & 71 & Prostate & & & & & \\
\hline 13 & LRS & M & 62 & Lung & Moderate KPS, Frankel C & None VIM, MOM, > 3 ESM, None VRM & L3, L4 & Desc & $10 / 23 / 2009$ \\
\hline 14 & RJF & M & 64 & Prostate & Bad KPS, Frankel D & None VIM, MOM, > 3 ESM, > 3 VRM & T6 & Desc & $11 / 04 / 2009$ \\
\hline 15 & JRA & M & 71 & & & & $\mathrm{~T} 7, \mathrm{~T} 8$ & & 02/04/2010 \\
\hline 16 & JBS & M & 56 & Prostate & Good KPS, Frankel E & None VIM, MOM, One ESM, Two VRM & C3, C4 & Desc + PS & $02 / 11 / 2010$ \\
\hline 17 & NP & M & 75 & Prostate & Bad KPS, Frankel B & None VIM, MOM, > 3 ESM, None VRM & T6 & Desc & $03 / 24 / 2010$ \\
\hline
\end{tabular}

KPS: Karnofsky Performance Status; De: Decompression (laminectomy); COR: Corpectomy; STTR: Subtotal Tumor Resection; PTR: Partial Tumor Resection; PS: Posterior Stabilization; AS: Anterior Stabilization; SEM: Spinal cord Epidural Metastasis; NTVM: Non-Treatable Visceral Metastases; VIM: Visceral Metastases; VRM:Vertebral Metastases; SOM: Solitary Osseous Metastasis; MOM: Multiple Osseous Metastases; ESM: Extra-Spinal Metastases.

Table 3. Results of three different prognostic score scales in 17 patients with spinal cord epidural metastasis, and correlation with their actual survival time. Results in bold indicate correct correlation between predicted survival prognosis and actual survival time, while the others results their incorrect correlation.

\begin{tabular}{lcccc}
\hline Patients & Actual & \multicolumn{3}{c}{ Total Scores } \\
\hline 1 & Survival Time & Tokuhashi & Tomita & Bauer \\
2 & Still alive, 22 months & 9 & 7 & 1 \\
3 & 3 months & $\mathbf{8}$ & 7 & 3 \\
4 & 2 months & 12 & 3 & 3 \\
5 & 9 months & 8 & $\mathbf{6}$ & $\mathbf{3}$ \\
6 & 1.5 month & 9 & $\mathbf{9}$ & 2 \\
7 & Still alive, 14 months & 9 & $\mathbf{4}$ & 2 \\
8 & Still alive, 27 months & 11 & $\mathbf{4}$ & 2 \\
9 & 8 months & $\mathbf{9}$ & 7 & 3 \\
10 & 2 months & $\mathbf{8}$ & 7 & 3 \\
11 & Still alive, 22 months & 11 & 6 & 2 \\
12 & 10 months & 8 & $\mathbf{6}$ & 2 \\
13 & 2 months & $\mathbf{8}$ & 4 & 2 \\
14 & 3 months & $\mathbf{6}$ & 7 & $\mathbf{1}$ \\
15 & 2 months & $\mathbf{8}$ & 4 & 2 \\
16 & 1 month & $\mathbf{4}$ & $\mathbf{8}$ & $\mathbf{0}$ \\
17 & 8 months & 13 & $\mathbf{4}$ & $\mathbf{2}$ \\
\hline
\end{tabular}

\section{Tomita score scale}

Tomita et al. ${ }^{4}$ designed another scoring system, not only to predict survival of SEM patients but also to recommend the most appropriate treatment. The Tomita Score ${ }^{4}$ consists of three prognostic factors: 1- grade of malignancy (slow growth, 1 point; moderate growth, 2 points; rapid growth, 4 points), 2- visceral metastases (no metastasis, 0 points; treatable, 2 points: untreatable, 4 points), and 3- bone metastases (solitary or isolated, 1 point; multiple, 2 points). These three factors are added together, and the final score ranges between 2-10 and is related to a treatment goal (Table 1).

Patients with a prognostic score of 2 or 3 have an expected survival longer than two years and SEM local control is recommended with wide or marginal excision of the SEM. Unfortunately, our only patient included in this score range died 2 months after surgery.

Patients scoring 4 or 5 points will probably survive about 2 years and intralesional surgical excision of SEM is recommended. Six patients were included in this category, but 4 (67\%) died before achieve this survival (mean actual survival of 6.5 months) and 2 (33\%) were still alive for more than one year.

Expected survival of patients with a prognostic score of 6 or 7 points was approximately 12 months in the Tomita original series and palliative surgery such as spinal cord decompression with stabilization was addressed as the first choice for short-term palliation. Eight patients in our study scored into this interval: 4 of them (50\%) died before 9 months, 2 (25\%) died between 9-12 months and 2 patients (25\%) were still alive 22 months after surgery.

Patients with 8, 9 or 10 points would be candidate only for supportive care, and not for surgical treatment, because they would probably live about 6 months. Two of our patients were included in this category and actually lived shortly (mean actual survival of only 1.25 months).

The Tomita scoring system accuracy to predict the survival, when applied in our series, was $0 \%, 33 \%, 25 \%, 100 \%$ for long-term, middle-term, short-term and supportive treatment, respectively. Note here the term "supportive treatment" is used for patients with a predicted survival of about 6 months, while the other score systems use the term 
"short-term" for these patients. If all categories are combined, the overall accuracy of this score was $35 \%$ (6 of 17 patients).

\section{Bauer modified score scale}

Bauer et al. ${ }^{9}$, and later modified by Leithner et al. ${ }^{12}$, divided the SEM patients according to the objective of their treatment and the most appropriate surgical strategy to be considered. Indirectly, the survival prediction time of each score range can be estimated. One point is given to each parameter: absence of visceral metastases, absence of lung cancer, presence of a solitary skeletal metastasis and primary cancer being breast, kidney, lymphoma or multiple myeloma. The sum of these factors varies from 0 to 4, and higher scores are related to better prognosis (Table 1).

Patients scoring 3 to 4 points should live longer (possibly about one year) and will benefit from local SEM control. Five patients were included in this score range, but only one (20\%) lived close to one year (9 months) and the others fours (80\%) died before. Their mean actual survival was 4.8 months.

Scores with 2 points includes the patient in the short-term survival and palliative surgical group, probably with a predicted survival close to 6 months. Nine patients (52\%) scored into this category: three of them died (33\%) before 3 months, one patient (11\%) survived 8 months and five patients (56\%) lived more than 9 months. Three of these patients were still alive at the end of follow-up.

Three patients were included in the 0 to 1 point score range, where the original authors ${ }^{9}$ indicate only supportive care. Two of them (67\%) died briefly after surgery (1 and 3 months, mean actual survival of 2 months) and the other (33\%) was still alive after 22 months of follow-up.

We estimated the Bauer modified score predicted survival as: approximately 12 months for the middle-term survival/local surgical control group (3 or 4 points), approximately 6 months for the short-term survival/palliative surgical group (2 points) and less than 6 months for the supportive care group (0 to 1 point). Accordingly, the accuracy for each group was $20 \%, 11 \%$ and $67 \%$, respectively. The overall Bauer modified score accuracy was $24 \%$.

\section{DISCUSSION}

Tokuhashi et al. ${ }^{6}$ studied his revised score retrospectively in 246 patients and the overall accuracy found was $82.5 \%$, noting it was higher than $78 \%$ for all categories individually. Tomita et al. ${ }^{4}$ reviewed 67 patients treated from 1987-1991 and reported a correlation of $69 \%$ between expected and actual patient's survival times ( $\mathrm{p}<0.0001)$. Leithner et al. ${ }^{12}$ modified the originally Bauer score ${ }^{9}$ and compared it to other six score scales studying 69 patients in Graz, Austria, concluding it was statistically significant able to predict survival.

Leithner et al. ${ }^{8}$ compared 7 preoperative prognostic scoring systems for spinal metastases in 69 patients in Graz,
Austria, including the ones we studied. A good prognostication with statistically significant results was provided for the three scores used in our study, with Bauer original and modified scores achieving the highest statistical significance.

Ulmar et al. ${ }^{13}$ compared the Tomita and Tokuhashi scores reliability to predict survival for 37 renal cancer patients with vertebral metastases in Ulm, Germany. They report reliable results with a statistically high significance for all categories combined or separately (higher than 54\%) when using the Tokuhashi Score Scale, but failed to show significant results for the prognosticated and real survival in these patients when using the Tomita Score.

All three scores systems demonstrated a low accuracy to predict patient's survival in our study. For Bauer modified and Tomita scores, we accepted a 6-month interval around the predicted survival time to indicate if a correct prognosis had been made. The Tokuhashi revised, Tomita and Bauer modified scoring systems had an overall accuracy of $41 \%$, $35 \%$ and $24 \%$, respectively. Unfortunately, statistical analyses couldn't be made due to the few number of patients enrolled.

A better accuracy to predict the survival in the short-term survival categories was observed: $75 \%, 100 \%$ and $67 \%$ of accuracy for Tokuhashi revised, Tomita and Bauer modified scores, respectively. However, a worse accuracy to predict the survival in the long-term survival score ranges was noted: $0 \%$, $0 \%$ and $20 \%$ of accuracy for Tokuhashi revised, Tomita and Bauer modified scores, respectively. The higher accuracy in predicting short-term survival is probably feasible because systemic metastatic disease, multiple organs failure and aggressive histological subtypes won't lead to long survival, but on the other hand the survival in less severely ill patients is affected by many other variables and can result in an unpredictable pattern of survival.

The accuracy to predict a middle-term survival varied between the different scores. Five of seven patients (71\%) with 9 to 11 points in the Tokuhashi revised score lived longer than expected, and only 1 patient (14\%) had a correct survival prediction. Fourteen patients scored 4 to 7 points in the Tomita Score, and 4 of them (25\%) died within the expected survival interval. Nine patients scored 2 points in the Bauer Modified Score, and only 1 (11\%) had a middle-term survival.

Every score system has important limitations and, although the accuracy of these scores to predict survival was seen with reasonable results in previous reports $[8,11]$, their low accuracy seen in our patients might be explained by differences between our population and the populations where the score scales were first invented. Our institution is located in São Paulo, an 18-million-people city in the Brazilian southeast and is known to receive all sorts of patients. Although São Paulo is a wealthy city, most of our patients are poor and, as this study was made retrospectively, the patients enrolled represent a general sample of SEM patients seen in our institution because none attempt the pre-select them to the study was made previously. 
Patients possibly take a longer time interval between the presentation of symptoms and the first consultation in our institution than would take in Tokyo, Kanazawa or Graz. Some factors might delay the patient to achieve the most appropriate treatment: our patients are usually seen by several doctors before being directed to a more specialized medical center, the low level of education of our population, and distance from their home town. Probably our hospital receives patients in a more advanced stage of their disease and most of them will have a shorter survival after SEM diagnosis. As a result, a long-term survival prediction will probably be incorrect at the time these patients are admitted in our hospital.
Retrospective analyses, few patients (total and within each primary cancer histology), patients lost during follow-up are important bias in this study.

In conclusion, our results can be seen as an alert that the score systems studied should be used in different populations with caution. More appropriate and reliable score scales need to be created or adapted to be suitable for our population, and we emphasize that predicting the patient survival wrongly can lead the medical staff to choose inappropriate treatments to each patient, and possibly decrease the overall survival.

\section{References}

1. Mut M, Schiff D, Shaffrey ME. Metastasis to nervous system: spinal epidural and intramedullary metastases. J Neurooncol. 2005;75(1):43-56. doi:10.1007/s11060-004-8097-2

2. Gilbert RW, Kim JH, Posner JB. Epidural spinal cord compression from metastatic tumor: diagnosis and treatment. Ann Neurol. 1978;3(1):40-51. doi:10.1002/ana.410030107

3. Hirabayashi H, Ebara S, Kinoshita T, Yuzawa Y, Nakamura I, Takahashi $J$ et al. Clinical outcome and survival after palliative surgery for spinal metastases. Cancer. 2003;97(2):476-84. doi:10.1002/cncr.11039

4. Tomita K, Kawahara N, Kobayashi T, Yoshida A, Murakami H, Akamaru T. Surgical strategy for spinal metastases. Spine. 2001;26(3):298-306. doi:10.1097/00007632-200102010-00016

5. Walker MP, Yaszemski MJ, Kim CW, Talac R, Currier BL. Metastatic disease of the spine: evaluation and treatment. Clin Orthop Relat Res. 2003;415 Suppl:S165-75.

6. Tokuhashi Y, Matsuzaki H, Oda H, Oshima M, Ryu J. A revised scoring system for preoperative evaluation of metastatic spine tumor prognosis. Spine. 2005;30(19):2186-91. doi:10.1097/01.brs.0000180401.06919.a5

7. Ribas ES, Schiff D. Spinal cord compression. Current treatment options in neurology. 2012;14(4):391-401. doi:10.1007/s11940-012-0176-7
8. Leithner A, Radl R, Gruber G, Hochegger M, Leithner K, Welkerling $\mathrm{H}$ et al. Predictive value of seven preoperative prognostic scoring systems for spinal metastases. Eur Spine J. 2008;17(11):1488-95. doi:10.1007/s00586-008-0763-1

9. Bauer HC, Wedin R. Survival after surgery for spinal and extremity metastases. Prognostication in 241 patients. Acta Orthop Scand. 1995;66(2):143-6. doi:10.3109/17453679508995508

10. Tokuhashi Y, Matsuzaki H, Toriyama S, Kawano H, Ohsaka $\mathrm{S}$, Scoring system for the preoperative evaluation of metastatic spine tumor prognosis. Spine. 1990;15(11):1110-3. doi:10.1097/00007632-199011010-00005

11. Tokuhashi Y. Letter to the editor. Spine. 2000;25(5):654. doi:10.1097/00007632-200003010-00022

12. Leithner A, Radl R, Gruber G, Hochegger M, Leithner K, Welkerling $\mathrm{H}$ et al. Predictive value of seven preoperative prognostic scoring systems for spinal metastases. Eur Spine J. 2008;17(11):1488-95. doi:10.1007/s00586-008-0763-1

13. Ulmar B, Naumann U, Catalkaya S, Muche R, Cakir B, Schmidt R et al. Prognosis scores of Tokuhashi and Tomita for patients with spinal metastases of renal cancer. Ann Surg Oncol. 2007;14(2):998-1004. doi:10.1245/s10434-006-9000-5 